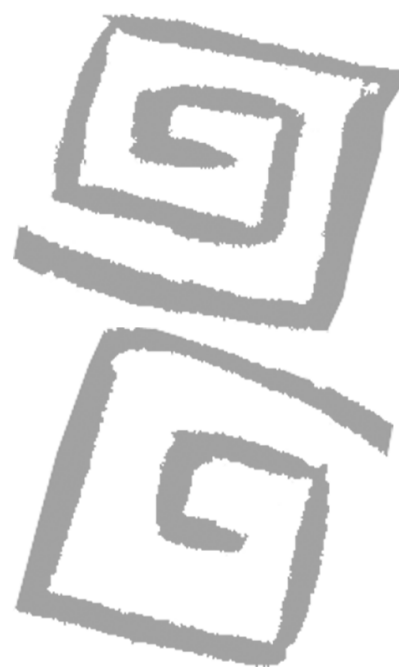

\title{
La comprensión de los significados de la maternidad: el caso de un programa de cuidado prenatal en un centro de salud en Popayán, Colombia
}

\author{
Understanding the meanings of motherhood: the case of \\ a prenatal care program in a health center in Popayán, \\ Colombia
}

Castro Franco, Bibiana Edivey ${ }^{1}$; Peñaranda Correa, Fernando ${ }^{2}$

${ }^{1}$ Psicóloga. Especialista en Psicología Social aplicada a la Efectividad Organizacional, Magíster en Salud Pública. Profesora, Universidad del Cauca, Colombia.

bibianacastro01@hotmail.com

${ }^{2}$ Médico. Doctor en Ciencias Sociales, Niñez y Juventud. Profesor asociado, Facultad Nacional de Salud Pública, Universidad de Antioquia, Colombia.

fernandopenaranda@gmail.com
RESUMEN El objetivo de este estudio es comprender los significados que sobre la maternidad tienen los actores sociales (mujeres embarazadas y personal de salud) dentro del programa prenatal de un centro de salud en la ciudad de Popayán, Colombia, programa implementado como estrategia para prevenir y manejar en forma oportuna las complicaciones obstétricas y perinatales. Para ello, se realizó una investigación cualitativa basada en entrevistas y observación de las actividades educativas colectivas. Entre los hallazgos, se encontraron discrepancias entre los actores sociales respecto de los significados de la maternidad, debido al conocimiento biomédico y a las relaciones de poder, aunque también vinculadas a los referentes culturales particulares. Estas discrepancias no reconocidas afectan la pertinencia del programa para responder a las necesidades y características socioculturales de las mujeres embarazadas.

PALABRAS CLAVE Salud Pública; Atención Prenatal; Maternidades; Investigación Cualitativa; Colombia.

ABSTRACT The objective of this study is to understand the meanings of motherhood for different social actors (pregnant women and health personnel) within the prenatal care program of a health center in the city of Popayán, Colombia, a program developed as a strategy for the prevention and treatment of obstetric and perinatal complications. In order to uncover these meanings, a qualitative research study was carried out using interviews with social actors and observation of collective educational activities. Discrepancies were observed among social actors about the meanings of motherhood, owing to the influence of biomedical knowledge and the differences in power relations, as well as to particular cultural referents. The lack of recognition of these discrepancies affects the program's capacity to respond to the needs and socio-cultural characteristics of the pregnant women it serves.

KEY WORDS Public Health; Prenatal Care; Hospitals, Maternity; Qualitative Research; Colombia. 


\section{INTRODUCCIÓN}

La atención de la mujer embarazada ha sido establecida como una prioridad de la salud pública por la Organización Mundial de la Salud (OMS) desde su constitución en 1948. Su ratificación, en Alma Ata en 1978, Ilevó al fortalecimiento de los programas de cuidado prenatal, dentro del denominado componente materno-infantil (1 p.3-12). Esta trascendencia ha sido materia de políticas, compromisos y legislaciones en los ámbitos internacional (2-10) y nacional $(11,12)$. Es así como la salud materno-infantil se ha convertido en un imperativo moral y político de los Estados (1).

Pero, tal como afirma la OMS en su Informe sobre la salud en el mundo 2005: ¡Cada madre y cada niño contarán! (1), a pesar de la relevancia antes anotada, persisten serios problemas en la atención que el sector salud provee a las mujeres embarazadas y sus familias, entre otros, las deficiencias para responder a las características culturales y necesidades de las mujeres y sus familias (1).

Una forma de explicar esta situación puede encontrarse en la antropología médica. Martínez (13) plantea que el modelo biomédico ha centrado la comprensión de la vida y la enfermedad en una perspectiva biológica desde la cual sus dimensiones sociales y culturales se reducen a causas secundarias o factores de riesgo. Es entendible, entonces, que los programas del componente materno-infantil hayan olvidado incorporar categorías como maternidad y crianza, cuya comprensión requiere un análisis ontológico, cultural, social e histórico (14).

Así, desde una perspectiva crítica y antropológica se reconoce la necesidad de estudiar la salud y la enfermedad teniendo en cuenta la biografía del individuo, las relaciones sociales y las representaciones culturales en un contexto histórico y económico-político particular (13).

Por lo tanto, se hace necesario ampliar la mirada con la que se estudian las acciones de los sistemas de salud, en este caso, el programa prenatal, el cual requiere ser comprendido como un hecho sociocultural. Ahora bien, si se entiende la cultura como esa red de significados en la cual los comportamientos de las personas se hacen inteligibles (15), será fundamental comprender los significados que tiene la maternidad para los actores del programa prenatal.

Pero para analizar los significados sobre la maternidad en un escenario biomédico es necesario reconocer que el programa prenatal constituye un terreno en el cual el personal de salud y las mujeres embarazadas se posicionan de manera diferente $y$, en ocasiones, de forma conflictiva, dado el poder que da el conocimiento biomédico a los primeros para definir los significados considerados como legítimos en dicho escenario (16-21). También se hace necesario analizar las diferencias respecto a los significados de la maternidad entre estos dos grupos pues, siguiendo a Bourdieu y Passeron (20) y a Bernstein (19), el discurso biomédico pedagógico se recontextualiza con el referente cultural o el arbitrio cultural (20) del agente institucional (21).

Este artículo es el resultado de una investigación denominada "Significados de la maternidad y de la educación en el embarazo para las mujeres embarazadas y el personal del programa prenatal, en el Centro de Salud del Suroccidente de la Empresa Social del Estado de Popayán, año 2009". Debido a la extensión de los resultados finales del estudio, el artículo abordará únicamente los hallazgos referidos a los significados que la maternidad tiene para las mujeres embarazadas y el personal de salud.

\section{MÉTODO}

Se llevó a cabo una investigación cualitativa que recibe una influencia de la etnografía, pero que toma diferentes técnicas de acuerdo a las necesidades, tal como lo plantean Denzin y Lincoln para el investigador considerado "bricoleur" (22). Así, se ha recogido de la etnografía su perspectiva de estudio "en" caso orientada a comprender el fenómeno en cuestión (los significados de la maternidad), teniendo en cuenta su carácter interpretativo y microscópico (15). Desde esta perspectiva, los hallazgos no pretenden generalizarse a otros grupos humanos y a otras situaciones, sino que más bien apuntan a la construcción de interpretaciones como construcciones teóricas que puedan servir para avanzar en la comprensión del fenómeno (15). 
Se seleccionó uno de los programas de cuidado prenatal de la Empresa Social del Estado de Popayán, Colombia, con base en los siguientes criterios: fortaleza del programa (desarrollo programático, organización, logística, trayectoria y experiencia del personal de salud) y disposición para participar en el estudio por parte de los integrantes.

El programa cuenta con siete médicos, dos enfermeras jefas y diez auxiliares de enfermería, personal que también apoya a los demás programas del centro de salud. Las sesiones de consulta ofrecidas a las mujeres embarazadas duran aproximadamente 15 minutos. Los casos diagnosticados como de riesgo los asumen los médicos como parte de su consulta diaria y los casos no complicados son atendidos por las enfermeras.

El trabajo de campo se llevó a cabo entre junio y octubre de 2009, lapso en el cual se hicieron 24 visitas al centro de salud. La recolección de la información se realizó por medio de entrevistas semiestructuradas grupales e individuales a las mujeres embarazadas y al personal de salud, y a través de la observación de algunas de las actividades del programa (por ejemplo, la toma de la muestra para el VIH, pues corresponde a la única actividad de educación grupal y la investigación estaba orientada también a comprender el proceso educativo).

En las entrevistas, se utilizaron guías de preguntas que abordaron los siguientes temas:

Con las mujeres embarazadas:

- los significados sobre el embarazo, la maternidad, el hijo o la hija por nacer y su relación con este/a;

- las relaciones con su pareja y su familia ampliada;

- los significados sobre el programa prenatal y su proceso educativo;

- las condiciones socioeconómicas de la mujer y su familia.

Con el personal de salud:

- los significados sobre el embarazo y la maternidad en general;

- los significados que, según ellos, tienen las mujeres embarazadas que atienden, acerca de su embarazo y maternidad;
- los significados que tienen acerca del programa prenatal y su proceso educativo.

Las guías de preguntas se fueron ajustando como resultado de los hallazgos y del proceso de análisis que se fue desarrollando concomitantemente con el trabajo de campo.

Al finalizar las actividades educativas relacionadas con la toma de las muestras para VIH, se invitó a las mujeres embarazadas a participar en la investigación. Con aquellas que quisieron participar se efectuaron un total de seis entrevistas grupales, con grupos de número variable, generalmente entre seis y ocho participantes. Algunas, que encontraron valiosa la experiencia, repitieron su participación, dado que se promovió un ambiente de discusión y reflexión colectiva que permitió a las mujeres compartir sus necesidades, emociones y angustias, en un ambiente de comprensión y respeto que sirvió como instancia de soporte. Con tres mujeres, interesadas en hablar sobre sus embarazos y aportar al estudio, se hicieron dos entrevistas individuales a cada una, después de las reuniones grupales o en otros momentos.

Con los siete médicos se hicieron dos entrevistas grupales y con cuatro de ellos entrevistas individuales. Con las dos enfermeras se realizaron una entrevista grupal y una entrevista individual con cada una. Finalmente, con las auxiliares de enfermería se llevaron a cabo tres entrevistas grupales (a la primera asistieron todas, a la segunda seis y a la última cuatro).

Participaron en total 34 mujeres embarazadas cuyas edades oscilaron entre los 18 y los 41 años, 18 eran multíparas y 16 primigestantes, con tiempos de embarazo que fluctuaron entre 10 y 30 semanas. Pertenecían a los estratos socioeconómicos 1 y 2 (población más pobre), los cuales corresponden a la población cubierta por el régimen subsidiado del Sistema de Salud. En cuanto a su estado civil, 18 eran madres solteras, 10 vivían en unión libre y seis estaban casadas. Respecto al nivel de educación formal, cuatro habían cursado la primaria, 27 contaban con algún grado de educación secundaria básica y tres tenían algún nivel de formación técnica o universitaria (ninguna completa).

Para el registro de la información, se utilizaron el diario de campo y la grabación de las 
entrevistas. Posteriormente se editaron las notas de campo y se transcribieron los materiales grabados. El proceso de análisis se realizó de manera simultánea con el de recolección de información, mediante la codificación y categorización de la información, basado en un proceso de comparación constante $(23,24)$. Las categorías se fueron construyendo teniendo en cuenta tanto los hallazgos como las preguntas centrales de la investigación. Con dichas categorías se orienta la presentación de los resultados en el presente artículo. Es importante anotar que los hallazgos presentados recogen la información proveniente únicamente de las entrevistas.

El proyecto de investigación fue aprobado por el Comité de Bioética de la Facultad Nacional de Salud Pública de la Universidad de Antioquia, lo cual consta en el acta 034 del 17 de junio de 2009 de ese organismo. Los participantes firmaron el consentimiento informado una vez que se les explicó el propósito de la investigación y decidieron participar voluntariamente. Se les anunció el carácter anónimo de la información suministrada por ellos y la posibilidad de abandonar el estudio en cualquier momento.

\section{RESULTADOS}

En primer lugar se presentan los significados de la maternidad para las mujeres embarazadas, relacionados con el comienzo del embarazo y su evolución, el cambio de identidad y el rol que asumen como madres, y las expectativas que tienen con relación a sus hijos/as y la crianza, y al parto. A continuación se presentan los significados que, según el personal de salud, tiene la maternidad para las mujeres embarazadas.

Para la gran mayoría de las mujeres entrevistadas, el embarazo fue percibido en sus inicios como sorpresivo e inoportuno. Lo encontraron como una novedad en sus vidas debido a los cambios físicos, emocionales y psicosociales. Se encontraron diferencias en cuanto a la aceptación del embarazo y su rol como madres. Algunas de ellas sintieron frustrados sus planes personales en la medida en que se vieron obligadas a abandonar su lugar de residencia, de trabajo, de estudios $y$, en general, sus expectativas de vida.
M9.-Yo tengo curiosidad, miedo, felicidad, nervios. Curiosidad al pensar cómo estará por dentro, cómo se estará formando; felicidad porque es mi primer embarazo; nervios por el parto. (30 semanas de embarazo)

M1.-Yo me he dedicado a mis hijos. El primero lo tuve a los 14 años y tenía la esperanza de que como ya estaban más grandecitos, iba a descansar un poco, para estudiar y trabajar, pero con el nuevo bebé, todo lo que ya había organizado se ha truncado. (10 semanas de embarazo)

Esta situación inesperada -y que significaba para ellas no estar preparadas- implicaba la necesidad de contar con tiempo para adaptarse a su nueva condición, y para imaginarse a sus hijos/as por nacer y su rol como madres, así como su futuro y el de ellos/as. Las mujeres no contemplaban una dedicación exclusiva a su rol como madres y expresaron que estaban construyendo una identidad como madres con base en sus propias historias, necesidades y proyectos de índole profesional, laboral y sentimental. En este sentido, manifestaron su expectativa de trascender dicho rol, visto como uno de los muchos proyectos que pensaban desarrollar, por lo que percibían algunas de las tareas de la crianza como impedimento para sus intereses personales.

\footnotetext{
M16.-Fue una sorpresa, pensé en abortar, luego le tomé cariño y empecé a imaginármelo, siento que él se mueve cuando le hablo. (20 semanas).

M10.-Yo no quiero seguir viviendo como mi mamá, que nos quiso tanto y dejó su vida. Yo quiero conocer otra persona, formar un hogar con él y mi hijo, seguir estudiando, y trabajar paralelamente. Mi mamá se entregó mucho a nosotros, dejó de vivir por nosotros. Yo le digo: "salga, consiga amigas". Yo sé que ella es feliz, pero le hace falta algo. (20 semanas)
}

También hablaron sobre las inquietudes y preocupaciones que los cambios generados como consecuencia del embarazo habían suscitado en ellas. Eran cambios en las relaciones con otras personas, especialmente con familiares, con sus parejas, con amigos y con el personal de salud. De la misma forma, manifestaron preocupación y angustia hacia su situación económica, 
ante las demandas que implicaba el embarazo y la crianza de un/a hijo/a. Esta situación las hacía sentir vulnerables y necesitadas de ayuda. Algunas madres expresaron la necesidad de realizar cambios en su estilo de vida durante el embarazo, que las llevara a concentrarse en sus hogares y a reducir el círculo de relaciones. Manifestaron igualmente cambios en su vida emocional, como incremento de la ansiedad y la depresión, que hacían más compleja su condición de inseguridad y vulnerabilidad. Por esto expresaron la necesidad de ser escuchadas en sus sentimientos y preocupaciones. Esperaban que se las considerara como un caso diferenciado con respecto a sus particularidades y necesidades específicas, por lo que demandaban un trato personalizado y cálido.

\footnotetext{
M18.-Aquí lo regañan a uno: "que el bebé tan chiquito, que tan descuidada". ¡Me asustan! No ven todo lo que he tenido que hacer para conseguir que me atendieran. (30 semanas)
}

Las mujeres que fueron abandonadas por sus parejas se mostraron abrumadas por su situación; para ellas y para las que contaban con su compañero, la pareja era fundamental como apoyo sentimental, afectivo, social y económico. Fue común en todas las entrevistadas la tendencia a experimentar, en este momento de sus vidas, la necesidad de apoyo por parte de sus madres, a quienes consideraron como un referente incondicional.

Por otro lado, encontraban que la responsabilidad del embarazo y del hijo/a por nacer era de ellas principalmente, aunque tuvieran la expectativa de contar con el apoyo de otras personas e instituciones para la crianza. Además, se atribuyeron una participación activa en la construcción de las relaciones del padre con el hijo.

M7.-Me dicen las amigas que no me ponga así [triste], porque el nene siente, que mi estado de ánimo lo afecta. Pero a mí me duele que el papá no lo reconozca, haber quedado en embarazo, pues yo me estaba cuidando. La enfermera me preguntó que por qué no me había cuidado, pero yo sí me estaba cuidando. (10 semanas)
Fueron muy variadas las expectativas hacia el/la hijo/a por nacer y hacia la función de crianza que esperaban cumplir. Algunas mujeres expresaron su temor a verse absorbidas por la crianza, por lo que esperaban fomentar la autonomía en el niño, de tal manera que no se diera un apego excesivo, por lo que deseaban compartir la crianza con otros familiares y las instituciones. Así, veían importante permitir la influencia del entorno social en el desarrollo moral y cognitivo de sus hijos. En este orden de ideas, no estaban inclinadas a realizar una lactancia prolongada y exclusiva.

\footnotetext{
M1.-...acostumbrarlo al teterito [biberón], y si uno tiene que salir, dejarlo con alguien. No solo conmigo. Así me pasó con mis primeros hijos: solo les daba seno y ningún otro alimento, y ellos se apegaron a mí y yo a ellos, y no me dejaban salir. (10 semanas)
}

En especial, para aquellas mujeres que estaban embarazadas por primera vez, la crianza de sus hijos constituía un asunto que les generaba angustia e incertidumbre, en la medida en que no se sentían preparadas. Les preocupaba no responder adecuadamente a las señales del recién nacido y el establecimiento de una buena relación primaria madre-hijo. De manera general, se mostraban inquietas y expectantes hacia la educación de el/la hijo/a y hacia su papel como educadoras. Consideraron que estas inquietudes sobre la crianza no habían sido abordadas por el personal de salud en el programa.

M24.-Yo me he puesto a pensar lo difícil que ha de ser darle de comer al bebé. Me pregunto: ¿qué voy hacer cuando llore? ¿Cómo serán los cuidados del bebé? ¿A qué horas he de cambiarlo? En los primeros días, mientras aprendo, me imagino que mi mamá y mi hermana me ayudarán. (24 semanas)

M22.-[Yo quisiera] que me enseñen cosas sobre el bebé, a estimularlo, a respirar en el parto, cosas que uno ignora. Yo he estado leyendo sobre estimulación al bebé. También mi familia me orienta con su experiencia, porque en el centro de salud, los médicos le responden lo que uno les pregunta, no le dan información adicional. (30 semanas) 
En un principio, el/la hijo/a por nacer se concebía como un extraño que afectaba la cotidianidad y los proyectos de la mujer, pero con la evolución del embarazo, esta percepción cambiaba a medida que este o esta se convertía en un ser sobre el cual se proyectaban expectativas y se construían imaginarios que eran fuente de una felicidad esperada.

La situación presentada se completa con un sentimiento de angustia y miedo hacia el parto, que se hacía más manifiesto al acercarse el nacimiento de el/la hijo/a. Esta sensación se veía exacerbada por la preocupación de poder recibir una atención del parto de buena calidad, pero también cálida y que respondiera a sus necesidades y condiciones particulares.

Las expresiones de las madres antes anotadas hacia la vivencia de sus embarazos evidenciaron posiciones ambivalentes, en la medida en que se cruzaban sentimientos de frustración, miedo e inquietud con manifestaciones y expectativas esperanzadoras hacia el/la hijo/a. Aun así, Ilama la atención que estas mujeres expresaron con mayor urgencia los sentimientos negativos que los positivos.

M5.-Las amigas dicen que el niño lo amarra [a uno] y eso me preocupa bastante. A mí me gustaría terminar el bachillerato y seguir adelante, pero a la vez me da miedo ocuparme de mis cosas y olvidarme de mi hijo. (20 semanas)

En las entrevistas realizadas al personal de salud se encontró que tienen un conocimiento general sobre las condiciones en que las mujeres asistentes al programa viven su embarazo. Asimismo, tienen sus propias percepciones respecto al tipo de sentimientos y apreciaciones que las madres embarazadas manifiestan sobre el embarazo y el hijo/a por nacer, y sobre las cuales expresaron sus opiniones. En algunos casos consideraban como inadecuada y perjudicial, para las mujeres embarazadas y su hijo/a por nacer, la posición de indiferencia y desmotivación que percibían en algunas de ellas, asunto que, según estas personas, requiere apoyo y tratamiento especializado por parte de otro personal de salud.

Uno sí se da cuenta que al tercer control se les ve a las embarazadas más alegres, más dispuestas; ya van reconociendo su embarazo, porque en el primer mes ellas no lo aceptan, pues se sienten mal. (Médico)

Uno dice que son madres desnaturalizadas, no tienen sangre para ser mamás [refiriéndose a quienes no expresan un sentimiento positivo hacia el embarazo]. (Auxiliar de enfermería)

Otras opiniones manifestaron su preocupación por la forma como las mujeres asumen el embarazo, en especial las adolescentes, a quienes consideraban poco responsables y poco comprometidas con la salud de su futuro hijo/a. En este sentido, percibían como perjudicial la condición de dependencia en la que estas mujeres jóvenes se situaban frente a sus madres.

Aunque con ciertos matices, en las expresiones del personal de salud se encontró una tendencia a concebir la maternidad desde una visión marcada por la exclusividad y la incondicionalidad. Se percibe en ellos un marcado énfasis en la dimensión biológica de la maternidad y el embarazo, así como un ideal de la maternidad como fundamento de la realización de la mujer, por lo que consideran que debe asumirse con compromiso y como prioridad.

\footnotetext{
Cuando veo dudosas a las embarazadas sobre el embarazo les digo: "la maternidad es una bendición de Dios, hay que tener confianza". Primero les pregunto si son creyentes. Es que una mujer nunca sabe qué es ser mujer, ni la felicidad, sino hasta el momento del parto. Se los explico así para que me entiendan: "que la felicidad de una mujer es el parto, tener el bebé, verlo sanito". (Enfermera)
}

Dicha prioridad se relacionó con la función de la reproducción y de la maternidad al servicio del bienestar y del cuidado de la salud física y emocional de el/la hijo/a. En este sentido, hubo una clara referencia a la mujer como última responsable de la salud y bienestar de el/la hijo/a. Así, se encontraron opiniones como que las mujeres son quienes deben asumir la responsabilidad final del cuidado del embarazo y, por lo tanto, en la participación en el programa de cuidado prenatal; o que los otros proyectos e ideales de la mujer no deben entrar en competencia con la maternidad: 
También me produce tristeza cuando una gestante es inasistente, o cuando las prioridades de ellas no son muy ubicadas: así tenga muchas aspiraciones, el bebé es la prioridad. (Enfermera)

Como una tendencia relevante en las entrevistas al personal de salud, se encontró que el/la hijo/a por nacer se percibe como vulnerable frente a las acciones de la mujer embarazada y de sus condiciones sociales y económicas. En este sentido, se lo concibe como un ser necesitado de protección y afecto por parte de sus futuras madres y se lo/a considera como prioridad de la mujer y como una responsabilidad para el personal de la salud.

Finalmente, otra tendencia observada es el reconocimiento que el personal de salud hace respecto a sus funciones relacionadas con la atención biomédica del embarazo, pero no así respecto a los asuntos emocionales y socioculturales del embarazo, la maternidad y la crianza, para lo cual manifestaron no disponer de tiempo, en la medida en que se considera materia fuera de su responsabilidad.

Yo, por el tiempo [falta], no les dedico lo que las embarazaditas necesitan. Ellas necesitan hablar de muchas cosas. Uno las ve angustiadas, preocupadas, deprimidas, pero yo las paro [cuando hablan sobre estos asuntos] o evito tocar el tema que las lleve a hablar. (Enfermera)

\section{DISCUSIÓN}

A continuación se presenta una propuesta que pretende avanzar en la comprensión de los hallazgos encontrados en este estudio. Para esto se utilizan aportes teóricos provenientes de la psicología social, la sociología y la antropología. Se ha estructurado en tres apartados. En el primero se analizan los significados de la maternidad en las mujeres embarazadas, en el segundo los significados de la maternidad en el personal de salud y en el tercero se particularizan algunos significados sobre la niñez y la maternidad que complementan el análisis realizado en los dos primeros apartados.

\section{Los significados de la maternidad en las mujeres embarazadas}

El embarazo en las mujeres, en particular si se trata del primer hijo, significa un cambio importante que implica la construcción de una nueva identidad y de transformaciones de orden social y económico $(25,26)$. Pero también implica la elaboración de una representación del niño/a por nacer y la construcción progresiva de un vínculo con él/ella $(25,27)$.

Según Stern (25), la madre requiere construir una "organización de la vida mental única, apropiada para hacerle frente a la realidad de cuidar un niño [...] denominada constelación maternal", como respuesta a las demandas de orden interior y exterior (socioeconómicas y culturales), especialmente a su necesidad de lograr que el/la hijo/a crezca y se desarrolle físicamente, de crear una matriz de apoyo que respalde su función y de reorganizar su identidad.

En cuanto a la pareja, el mismo Stern (25) plantea que la Ilegada de el/la hijo/a altera la red de modelos de la mujer sobre su pareja, en tanto padre, amante, marido y hombre. Por otro lado, Videla (28) señala que el embarazo afecta y se ve afectado por la relación de la madre con la familia extensa, debido a la complicada red de sentimientos que se establecen en el seno del núcleo familiar.

Al inicio del embarazo, lo económico, lo social y los proyectos de vida propios son aspectos que ocupan un lugar prioritario en las mujeres, mientras el/la hijo/a por nacer tiene una existencia marginal. Esto resulta así porque, para las madres, los primeros factores mencionados son evidentes y parten de la conciencia de sí misma (29), a diferencia de el/la hijo/a por nacer, que para muchas, solo lo confirma un examen de laboratorio.

Al respecto, Stern (25) plantea que la conciencia sobre la existencia de el/la futuro/a hijo/a se fortalece a partir de los cuatro meses de gestación, como se evidenció en las mujeres embarazadas del presente estudio, en la medida en que el feto crece y se desarrolla en el útero de la madre. Esto se corresponde con el período de gestación en el que se encontraban las entrevistadas, cuando empezaron a manifestar con mayor claridad sentimientos favorables hacia el embarazo. 
Lo anterior puede explicarse en función de que alrededor de las veinte semanas de gestación, las mujeres han contado ya con mayores oportunidades para interactuar con el/la futuro/a hijo/a y hacerlo parte de sí (25). La marginalidad de ese/a hijo/a por nacer en las primeras semanas va dando paso a una representación más amplia y viva de este/a, la cual también es producto de una expresión narcisista de las futuras madres con respecto a la realización de algunos de sus anhelos insatisfechos a través de el/la hijo/a $(25,30)$. En este sentido, requieren desarrollar una identificación positiva con su hijo/a para que puedan experimentar satisfacción frente a su existencia y ello implica tiempo.

En este sentido, Bettelheim (31) plantea las dificultades que representa para el ser humano asumir cambios en su vida, en la medida en que se afectan la identidad y la natural resistencia que se suele presentar ante ello, como defensa narcisista. Esta situación se encuentra mediada por la relación que logre establecer con las figuras maternales de la matriz de apoyo (25), las relaciones con el padre de el/la hijo/a, y demás condicionantes de su situación socioeconómica (28). Todo este proceso se da en el marco de profundas transformaciones socioculturales con respecto a la familia y a los roles de la mujer en la sociedad (32).

Así, la reacción inicial de rechazo de las madres frente al embarazo responde, precisamente, al mencionado proceso de reorganización identitaria, en el que la mujer entra en conflicto con sus prioridades o se encuentra en situaciones en las que el embarazo afecta sus condiciones socioeconómicas y afectivas, así como sus proyectos de vida o, simplemente, en que la maternidad no es esperada (28).

Adicionalmente, los hallazgos muestran que la dependencia de las figuras maternales, especialmente de sus madres, está presente en las entrevistadas, más allá de que sean adultas o adolescentes. Sin embargo, es más evidente en las últimas, probablemente por percibirse más vulnerables e inseguras consigo mismas y con su nuevo rol. Algunos autores señalan la importancia del modelo de relación con la madre en la construcción de las fantasías, esperanzas y temores de la mujer embarazada consigo misma y con su hijo $(25,26)$.
Desde esta perspectiva, se entienden entonces los sentimientos ambivalentes que las madres expresan ante el embarazo: interés y gusto por ser madres, pero también frustración, tristeza y malestar frente a los síntomas y limitaciones del embarazo, y exigencias en relación con la crianza.

Al respecto, Bettelheim (31) plantea la existencia de sentimientos contradictorios frente a los hijos por parte de los padres, al igual que altibajos en la relación, señalados como parte de la dinámica de vivencia familiar, y que se logra regular en la medida en que los padres realicen procesos identificatorios con los/las hijos/as.

Además de lo anterior, tal como se encontró en este estudio, un aspecto relevante lo constituye el sentimiento de inseguridad con respecto a la crianza, el cuidado y la educación de el/la hijo/a, al confrontar su realidad, sus conocimientos y su grado de preparación con sus expectativas o las de la sociedad, en lo referente a las competencias que le demanda su rol materno $(25,26,28)$.

Como consecuencia de este proceso de construcción identitaria, en un contexto de vulnerabilidad afectiva y socioeconómica, las mujeres anhelan y buscan el acompañamiento de la familia, la pareja y el personal de salud para ejercer la maternidad. La madre espera que esta matriz de apoyo la valore, la aprecie, la instruya y la ayude en su función de crianza $(25,32)$.

Ahora bien, la posición asumida por algunas mujeres entrevistadas -referidas a su intención de cuidarse, concentrarse en el hogar y disminuir su círculo de relaciones socialespuede entenderse como un repliegue sobre sí misma, como manera de fortalecerse y asumir los cambios que se vienen. Al respecto, Bettelheim (31) plantea que, frente a los grandes cambios que el ser humano experimenta a lo largo de la vida, necesita períodos de calma y de actividad para lograr un desarrollo satisfactorio. El ensimismamiento -que externamente puede confundirse con pasividad- puede darse cuando, dentro de la persona, se producen procesos internos de tal importancia que la abruman y le restan energía suficiente como para llevar a cabo acciones dirigidas al exterior. La quietud y la actividad son recursos con los que se aprende a comprender y a dominar tanto el mundo interno como el externo. 
Es así como las entrevistadas esperan lograr un equilibrio entre su necesidad de conservar su individualidad y las demandas que la sociedad les plantea para el cumplimiento de su rol de madre $(16,25,26,33)$. Sin embargo, dicho equilibrio es inestable porque siempre están cambiando los conflictos a los que tienen que hacer frente, y en la búsqueda de ese equilibrio tendrán que hacer concesiones que afectarán su proceso de reestructuración identitaria (34). Los resultados de este equilibrio dependerán de sus condiciones personales, la matriz de apoyo y su situación sociocultural.

\section{Los significados de la maternidad en el personal de salud}

El personal de salud también tiene, por su parte, conocimientos sobre los cambios y la posición de las mujeres hacia su embarazo y la maternidad, así como sobre los sentimientos, expectativas y tribulaciones que ellas expresan. Si dichas posiciones y sentimientos no concuerdan con sus conocimientos, concepciones y valores, comienzan a cuestionarlos, y los rotulan como problemas inconscientes que dificulta a las mujeres asumir la maternidad (28). En este sentido, si bien el personal de salud reconoce la influencia de la red de apoyo familiar (especialmente la pareja y la madre) sobre el estado de ánimo y la actitud de la mujer embarazada, fundamentalmente perciben su participación en el programa como mecanismo para lograr la homogeneización de conocimientos en el seno de la familia, de tal forma que no interfieran con el saber que el personal de salud busca promover.

La actitud de censura por parte del personal de salud responde a su dificultad para diferenciar la procreación como hecho biológico, de la maternidad como fenómeno social y personal en la cual inciden factores contextuales (32,35). Así, el profesional de la salud se acerca a la comprensión de la situación de las mujeres embarazadas desde una perspectiva biomédica que tiende a su homogeneización, en la medida en que el modelo biomédico se configura como un subuniverso de significados (16) que lo afecta poderosamente en sus concepciones y sus acciones, marcado por una orientación normalizadora, intervencionista, positivista $(36,37,38)$ y medicalizadora $(37)$.

Pero, además, los propios valores culturales del personal de salud se plantean como parámetros científicos de evaluación en la medida en que dicho profesional no es consciente de la influencia cultural en la construcción de su discurso educativo $(19,20,35)$. Por esto, valora en la mujer embarazada su identidad como madre y, aunque identifique otros roles e identidades en ella, no los reconoce como legítimos. Se mantienen entonces los modelos de familia y de roles maternales y paternales tradicionales, fortalecidos por los discursos disciplinarios (especialmente de la psicología y la sociología), religiosos y políticos que conciben a la mujer desde su función de crianza de los hijos y como principal y última proveedora de amor y cuidados para el niño $(35,39)$, basados en principios patriarcales y en una perspectiva biologicista que asocia la función reproductiva con la maternidad $(32,39)$. Por esto, los asuntos relacionados con el bienestar emocional y las condiciones socioeconómicas de la mujer embarazada se encuentran soslayados por la preeminencia de la atención biomédica, pero también porque se considera normal que las mujeres sacrifiquen su bienestar por el bienestar de su hijo/a (35).

De esta manera, el personal de salud, desde su función educadora, afecta la construcción identitaria de la mujer, sin que tenga una conciencia clara del fenómeno. En este sentido, Todorov (40) plantea que es deshumanizante negarles a las personas la posibilidad de ampliar su identidad, de tal manera que quede restringida a una única función.

Por otro lado, las inquietudes de las mujeres embarazadas con respecto a su competencia maternal y a su función de crianza, así como con respecto a su relación primaria con su hijo/a, no son atendidas por el personal de salud, debido a que dicho personal no prioriza esos aspectos como parte de sus funciones en el programa. Esta situación obedece a la perspectiva fragmentada del discurso biomédico, que soslaya las dimensiones socioculturales en la atención sanitaria (13) y dificulta la incorporación de categorías como maternidad y crianza para la estructuración de las actividades educativas en el programa (41). 


\section{La entronización del niño y los nuevos significados de la maternidad}

Lo descrito hasta aquí requiere de un análisis final en relación con tres aspectos socioculturales que ayudan a comprender la situación analizada en los dos apartados anteriores: la entronización de el/la niño/a, la maternidad como dimensión no natural en la mujer y los nuevos significados de la maternidad.

La entronización de el/la niño/a en la sociedad lo ubica como centro de la familia, a partir del cual la mujer adquiere un lugar de reconocimiento al instalarse como la principal proveedora de amor, cuidados y recursos económicos para el/la hijo/a. Así, se le ha impuesto a la mujer la responsabilidad principal sobre la salud física y emocional de los/las hijos/as, como también sobre la educación inicial, en un contexto de sobrevaloración de la niñez $(25,35,39)$.

Los sentimientos ambivalentes en relación con la maternidad encontrados en esta investigación resultan relevantes porque reflejan cómo la maternidad no forma parte de la naturaleza de la mujer y cuán difícil resulta asumirla para algunas de ellas, en la medida en que el embarazo y la maternidad están relacionados con las condiciones personales y socioculturales $(25,33,35,42-44)$.

Las manifestaciones de algunas entrevistadas, respecto a la posición crítica hacia la maternidad que asumieron sus madres o ellas mismas en el pasado, muestran una tendencia de cambio con respecto al significado que la maternidad tiene hoy para las mujeres. Actualmente, las mujeres están dándole relevancia a otros aspectos de su vida y no solo se están identificando en función de sus hijos/as; es decir, están construyendo un significado de maternidad no centrado en el esquema de sacrificio-entrega incondicional con respecto al/a la niño/a, y están intentando rescatar a la mujer como ser humano. De esta manera, las posibilidades de vivencia de la maternidad no se agotan en una única concepción; por el contrario, lo que esta investigación muestra es la pluralidad de significados que la maternidad tiene para las mujeres hoy en día, significados por demás complejos y paradójicos, algunos de los cuales pueden entrar en conflicto con los significados que se plantean desde el discurso biomédico.

\section{CONCLUSIONES}

En esta investigación se encontraron diferencias importantes en los significados de la maternidad entre las mujeres embarazadas y el personal de salud, reconociendo a su vez heterogeneidad al interior de cada uno de estos grupos. Esta diferencia está medida fundamentalmente por el conocimiento biomédico, aunque también entren en juego los referentes culturales particulares. Se requiere entonces, por parte de los sistemas de salud y del personal de salud, un mejor conocimiento de estos aspectos para diseñar y realizar políticas, programas y acciones más pertinentes y constructivas en relación con las necesidades y las características socioculturales de las mujeres embarazadas y sus familias. Lo anterior implica, por lo tanto, la construcción de ambientes y posiciones de respeto hacia la diversidad, en un mundo cada vez más plural.

El sector salud podrá apoyar mejor a las mujeres embarazadas y sus familias en esta función, como se desprende de esta investigación, si puede acceder a una perspectiva más amplia, más transdisciplinaria y más comprensiva. Es necesario también comprender que el desarrollo de los/las niños/as no puede hacerse a expensas de las mujeres; más aun, el desarrollo de ellos/as pasa por el desarrollo de los adultos que cumplen funciones de crianza. Entonces, los programas de cuidado prenatal deben preguntarse cómo hacer para lograr que las mujeres reciban una atención del embarazo, el parto y el puerperio con mejor calidad, no solo desde los indicadores biomédicos, sino también en el sentido de apoyar de la mejor manera su proceso de construcción identitaria y de preparación para su función de crianza, como ellas lo demandan.

Se considera prioritario desarrollar una visión comprensiva de la maternidad, en la que se aborden sus condicionantes socioculturales, y se la comprenda en relación con sentimientos y significados sociales implicados en la idea de ser madre, esto es, una noción de maternidad resultante de la actividad selectiva e interpretativa que las personas 
hacen en un contexto determinado, y no como inherente a la naturaleza de las mujeres (45).

Así, pues, trascender una visión homogeneizadora y normalizadora requerirá propuestas alternativas que reconozcan la inclusión de las categorías maternidad y crianza como complejos históricos, ontológicos y socioculturales y como temáticas centrales de los programas y acciones de atención a las mujeres embarazadas y a sus familias.

\section{REFERENCIA BIBLIOGRÁFICA}

1. Organización Mundial de la Salud (OMS). Las madres y los niños son importantes - y su salud también. En: Informe sobre la salud en el mundo 2005 [Internet]. 2005 [citado 7 feb 2011] p. 1-21. Disponible en: http://www.who.int/whr/2005/03 chap1_es.pdf

2. Naciones Unidas. Los objetivos de Desarrollo del Milenio [Internet]. Nueva York: Naciones Unidas; 25 de septiembre del 2008 [citado 7 de febrero del 2011]. Disponible en: http://www.un. org/spanish/millenniumgoals/pdf/MDGOverviewS PANISH.pdf

3. Naciones Unidas. Declaración Universal de los Derechos Humanos. [Internet]. 1948 [citado 7 feb 2011]. Disponible en: http://www.un.org/es/documents/udhr/

4. Organización Internacional del Trabajo. Convenio sobre la protección de la maternidad, 2000 [Internet]. Ginebra: OIT; 2000 [citado 7 feb 2011]. Disponible en: http://www.ilo.org/ilolex/ cgi-lex/convds.pl?C183

5. Oficina del Alto Comisionado de las Naciones Unidas para los Derechos Humanos. Convención sobre la eliminación de todas las formas de discriminación contra la mujer [Internet]. 1981 [citado 7 feb 2011]. Disponible en: http://www2.ohchr.org/ spanish/law/cedaw.htm
6. Oficina del Alto Comisionado de las Naciones Unidas para los Derechos Humanos. Convención sobre los Derechos del Niño [Internet]. 1989 [citado 7 de febrero del 2011]. Disponible en: http://www2.ohchr.org/spanish/law/crc.htm

7. Naciones Unidas. Cumbre Mundial en Favor de la Infancia [Internet]. 1990 [citado 7 feb 2011]. Disponible en: http://www.unicef.org/spanish/specialsession/about/world-summit.htm

8. Fondo de Poblaciones de las Naciones Unidas. Conferencia Internacional sobre la Población y el Desarrollo [Internet]. 1994 [citado 7 feb 2011]. Disponible en: http://www.senado.gob.mx/comisiones/LX/equidadygenero/content/marco/docs/10.pdf

9. Naciones Unidas. Informe de la Cuarta Conferencia Mundial sobre la Mujer [Internet]. Beijing; 1995 [citado 7 feb 2011] A/CONF.177/ 20/Rev.1. Disponible en: http://www.eclac.cl/ mujer/publicaciones/sinsigla/xml/3/6193/Platafor ma.pdf

10. Naciones Unidas, Comité de Derechos Económicos, Sociales y Culturales. Observaciones finales del Comité de Derechos Económicos, Sociales y Culturales: Israel [Internet]. 2001 [citado 7 feb 2011]. Disponible en: http://www.acnur. org/biblioteca/pdf/1620.pdf

11. República de Colombia. Decreto 3039 de 2007. Plan Nacional de Salud Pública 20072010. Bogotá: Diario Oficial 46716; 2007. 
12. Ministerio de la Protección Social. Maternidad segura. En: Política de Salud Sexual y Reproductiva. Bogotá: Fondo de Población de las Naciones Unidas; 2003. p. 29-33.

13. Martínez A. Antropología médica. Teorías sobre la cultura, el poder y la enfermedad. Barcelona: Anthropos; 2008.

14. Bastidas M, Torres N, Arango A, Escobar G, Peñaranda F. La comprensión de los significados que del programa de crecimiento y desarrollo tienen sus actores: un paso hacia su cualificación. Ciência \& Saúde Coletiva. 2009;14(5):1919-1928.

15. Geertz C. Descripción densa: hacia una teoría interpretativa de la cultura. En: Bohannan P, Glazer M, editores. Antropología: lecturas. 2a ed. Madrid: McGraw Hill/Interamericana; 1993. p. 547-568.

16. Berger $P$, Luckmann T. La construcción social de la realidad. Buenos Aires: Amorrortu; 1978.

17. Bernstein B. Poder, educación y conciencia: sociología de la transmisión cultural. Barcelona: El Roure; 1990.

18. Bernstein B. La estructura del discurso pedagógico. Clases, códigos y control. Madrid: Morata; 1993.

19. Bernstein B. Hacia una sociología del discurso pedagógico. Bogotá: Cooperativa Editorial Magisterio; 2000.

20. Bourdieu P, Passeron J. La reproducción. Elementos para una teoría del sistema de enseñanza. México DF: Fontamara; 1998.

21. Peñaranda F. Las fuerzas sociales y el escenario comunicativo en la educación para la salud: el caso del programa de crecimiento y desarrollo. Ciência \& Saúde Coletiva. 2011;16(10):4023-4031.

22. Denzin N, Lincoln Y. Introduction. The discipline and practice of qualitative research. En: Denzin $\mathrm{N}$, Lincoln $\mathrm{Y}$, editors. The Sage handbook of qualitative research. Thousand Oaks: Sage; 2005. p. 1-32.

23. Woods P. La escuela por dentro. La etnografía en la investigación educativa. Barcelona: Paidós; 1987.

24. Wolcott $H$. Transforming qualitative data: description, analysis and interpretation. Thousand Oaks: Sage; 1994.

25. Stern D. La constelación maternal. Un enfoque unificado de la psicoterapia con padres e hijos. Barcelona: Paidós Ibérica; 1997.
26. García A, De Cássia R. Representações de maternidade de mães jovens e suas mães. Psicología em Estudo. 2003;8 (num. esp.):63-73.

27. Canaval G, Gonzales M, Tovar M, Valencia C. La experiencia de las mujeres gestantes: "lo invisible". Investigación y Educación en Enfermería. 2003;21(2):32-46.

28. Videla M. Maternidad: mito y realidad. Buenos Aires: Nueva Visión; 1990.

29. Guelbenzu J. Signos de identidad. Glosario para una sociedad intercultural. Valencia: Bancaja; 2002.

30. Tenorio M. Instituir la deuda simbólica. Revista Colombiana de Psicología. 1993;(2):89-95.

31. Bettelheim B. Psicoanálisis de los cuentos de hadas. Barcelona: Crítica; 1984.

32. Puyana $Y$, compiladora. Padres y madres en cinco ciudades colombianas: cambios y permanencias. Bogotá: Almudena Editores; 2003.

33. Molina M. Transformaciones históricas culturales del concepto de maternidad y sus repercusiones en la identidad de la mujer. Psikhe. 2006;15(2):93-103.

34. Peñaranda F, Bastidas M, Escobar G, Torres N, Arango A, Pérez F. Educación para la salud: una mirada alternativa al modelo biomédico. La praxis como fundamento de una educación dialógica. Medellín: La Carreta; 2011

35. Badinter E. ¿Existe el instinto maternal? Historia del amor maternal. Siglos XVII al XX. Madrid: Paidós; 1991.

36. Lolas F. Más allá del cuerpo. La construcción narrativa de la salud. Santiago: Andrés Bello; 1997.

37. Foucault M. Historia de la medicalización. Educación Médica y Salud. 1977;(11):3-25.

38. Achard P. Discurso biológico y orden social: crítica de las teorías biologistas, psicología y ciencias sociales. México: Nueva Imagen; 1980.

39. Tenorio $M$, Ocampo $R$, Collazos $T$. Documento temático: Familia. Memoria del curso Familias y Culturas. Cali: Instituto de Psicología, Universidad del Valle: 2004.

40. Todorov T. Destinos de la identidad. Letras Libres [Internet] 2001 [citado 25 jun 2011];(nov). Disponible: http://www.letraslibres.com/revista/ convivio/destinos-de-la-identidad 
41. Peñaranda F, Bastidas M, Escobar G, Torres J, Arango A. Análisis integral de las prácticas pedagógicas de un programa educativo en Colombia. Salud Pública de México. 2006;48(3):229-235.

42. Palomar C. Maternidad, historia y cultura. La Ventana. 2005;22:35-67.

43. Tenorio M. Pautas y prácticas de crianza en familias colombianas. Bogotá: Ministerio de Educación Nacional y OEA; 2000.
44. Tenorio M. Amor materno y muerte infantil: En el nordeste brasileño de Nancy ScheperHughes. En: Documento temático para el curso Familia y Cultura. Cali: Instituto de Psicología, Universidad del Valle; 2004.

45. Castilla M. La ausencia del amamantamiento en la construcción de la buena maternidad. La Ventana. 2005;22:189-218.

\section{FORMA DE CITAR}

Castro Franco BE, Peñaranda Correa F. La comprensión de los significados de la maternidad: el caso de un programa de cuidado prenatal en un centro de salud en Popayán, Colombia. Salud Colectiva. 2011;7(3):333-345.

Recibido el 4 de abril de 2011

Versión final presentada el 17 de julio de 2011

Aprobado el 9 de septiembre de 2011 